\title{
Melanin Quantification by Chemical Methods in Healthy and Melanoma Cell Lines Correlated with the Importance in the Development of Melanoma
}

\author{
TIBERIU BRATU ${ }^{1 \#, ~ M I R C E A ~ B E R C E A N U ~}{ }^{2 \#, ~ D O R I N A ~ C O R I C O V A C ~}{ }^{2 *}$, \\ ZORIN CRAINICEANU ${ }^{1 *}$, ZSOLT GYORI ${ }^{1}$, ANDREEA ROMAN ${ }^{1}$, \\ IASMINA MARCOVICI ${ }^{2}$, CRISTINA DEHELEAN ${ }^{2}$, IULIA PINZARU ${ }^{2}$ \\ ${ }^{1}$ Victor Babes University of Medicine and Pharmacy Timişoara, Faculty of Medicine, Department of Plastic surgery, \\ 2 Eftimie Murgu Sq., 300041 Timisoara, Romania \\ ${ }^{2}$ Victor Babes University of Medicine and Pharmacy Timisoara, Faculty of Pharmacy, Department of Toxicology and \\ Drug Industry, 2 Eftimie Murgu Sq., 300041 Timisoara, Romania
}

Abstract: In the last decade, the incidence of skin cancer has increased significantly and there is data that rank it as the most common form of malignancy in humans. Among the classified skin cancers, melanoma is the most severe with a significant mortality rate. The determining factor in the occurrence of skin cancer is generally recognised to be ultraviolet radiation. Skin pigmentation has been shown to provide a high degree of protection and this is due to melanin. In order to be able to consider certain hypotheses and to develop new approaches to melanoma, in this study it was studied the melanin content in certain cell types that have a direct connection with melanoma. The cell lines used in the study were: (i) human melanoma cell lines: A375, SK-MEL-1, SK-MEL-3, SK-MEL-5, SKMEL-28, SH-4, and COLO 829; (ii) mouse melanoma cell lines: B164A5, B16FO, and B16F10; and (iii) healthy cell lines of human origin: HEMa (primary melanocytes), HaCaT (immortalised keratinocytes) and 1BR3 (human fibroblasts). The results obtained pointed out that A375, SK-MEL-28 and SK-MEL-5 cells do not produce melanin, SK-MEL-1, SH-4 and SK-MEL-3 cells produce melanin while COLO 829 cells - only in small passages produce melanin. Of the 3 lines of murine melanoma tested, the one that produces melanin in a higher concentration is B16F10, followed by B16FO and B164A5.

Keywords: melanin, content, cells, skin, melanoma

\section{Introduction}

Unique and specific, melanocytes - cells located in the basal layer of the epidermis - produce melanin that is deposited in melanosomes (membrane-bound organs) and then transferred to neighbouring keratinocytes. Melanin granules, after reaching keratinocytes, accumulate above the cell nuclei having the role of protecting deoxyribonucleic acid (DNA) from damage caused by ultraviolet radiation. Therefore, the function of skin pigmentation exerted by the "unit of epidermal melanin" is of two types: constitutive pigmentation in its basal state and optional pigmentation in response to environmental stimuli. [1] Among the markers described for the involvement of melanogenesis induced by ultraviolet radiation are transcription factors (MITF and SOX9), various melanosomal enzymes (tyrosine kinase TYR, tyrosinase 1 protein and DCT tyrosinase 2 protein). [2,3] In case of sun exposure, the skin is subjected to the tanning process, a process that varies significantly depending on its type, shape, and duration of exposure. Depending on the wavelengths of ultraviolet radiation, specific mechanisms are triggered that differentially modulate melanocyte-specific markers, with a synergistic effect on melanogenesis when combining type A and type B UV radiation [3,4]. Diseases such as skin whitening and vitiligo, which are associated with a poor-quality life of patients from a cosmetic point of view but also susceptibility to skin cancer, are due to melanocyte dysfunction [5]. 
It is well known that ultraviolet radiation causes reversible or irreversible damage to the skin leading to certain diseases. Of these diseases, in people with light skin, the most common is skin cancer and the main triggering factor is excessive exposure to the sun (excess of ultraviolet radiation). The association between the appearance of malignant skin diseases and its pigmentation was made several years ago, and currently the skin's response to sunlight is causally related to the rate and incidence of melanoma. The variation of melanin content in the skin is very wide, considering the skin types, so that the incidence of melanoma is higher in the poorly pigmented persons compared to the intensely pigmented ones. [6] The risk of melanoma is correlated with the individual characteristics of the pigment: it is higher in people with light skin colour, in those with red hair, with blue eyes, in those who have suffered sunburns throughout their lives. The main environmental factor responsible for the damage is the type B ultraviolet radiation $(280-320 \mathrm{~nm})$ due to the production of pyrimidine dimers, the cross-linking of the nucleoproteins breaks of the DNA chains, etc. These radiations trigger not only the appearance of melanoma but also other types of skin cancers (basal cell carcinoma, sarcomas, and squamous cell carcinomas of the skin) but by mechanisms different from those that induce melanoma. [6] Some categories of people are more susceptible to the development of the disease, for instance: (i) older people that live in areas with increased UV radiation exposure (due to a depleted ozone layer), another risk factor being the existence of co-morbidities (e.g. asthma, hypertension, etc.), and (ii) people with dental implants due to the composition of implants and materials used that might interact with the therapy, although the risk is minimal according to the new guidelines regarding tissue healing. $[7,8]$ Another melanoma-related pathology also common in adults is malignant choroidal melanoma, a primary intraocular malignancy, but with a lower risk of occurrence to persons that consume some natural plants (e.g. garlic or mistletoe), data supported by the scientific literature. [9] In recent years, a variety of compounds and formulations (especially nano-type) have been selected for both safety assessment and for establishing doses that are effective in addressing melanoma cells. [10-12]

Even though it is well known the origin of cutaneous melanoma (from mature melanocytes) [13], there are some uncertainties regarding the nature and number of cells with the ability to propagate tumours. To a certain extent, this is because the characterization of tumorigenic subsets purified by cancer cells has not been fully realized so far. In the present case, it is necessary to quantify melanin in the chosen lines due to the fact that the study and testing of new therapies is done in a first phase along these lines, after which we use 3D cells and in vivo studies.

The main aim of the current research study is to quantify the melanin content in seven human melanoma cell lines, three murine melanoma cell lines and three healthy human skin-related cell lines, since it was stated that melanin might interfere with melanoma therapies, like radio-, photo- and chemotherapy. [13]

\section{Materials and methods}

In the present research study were chosen: seven human melanoma cell lines - A375 (ATCC® CRL-1619 ${ }^{\mathrm{TM}}$ ), SK-MEL-1 (ATCC® ${ }^{\text {HTB-67TM}), ~ S K-M E L-3 ~(A T C C ® ~ H T B-69 T M), ~ S K-M E L-5 ~}$ (ATCC® HTB70 ${ }^{\mathrm{TM}}$ ), SK-MEL-28 (ATCC ${ }^{\circledR}$ HTB72 ${ }^{\mathrm{TM}}$ ), SH-4 (ATCC® CRL-7724TM) and COLO829 (ATCC® CRL-1974 ${ }^{\mathrm{TM}}$ ); three murine melanoma cell lines - B16-F0 (ATCC® CRL-6322 ${ }^{\mathrm{TM}}$ ), B16-F10 (ATCC® CRL-6475 ${ }^{\mathrm{TM}}$ ), and B164A5 (ECACC 94042254) and three skin related healthy human cell lines - HEMa (ATCC® PCS-200-013 ${ }^{\mathrm{TM}}$ ), HaCaT (CLS 300493) and 1BR3 (ECACC 90011801). The cell lines were purchased as frozen vials from American Type Culture Collection (ATCC), European Collection of Authenticated Cell Cultures (ECACC) and CLS Cell Lines Service $\mathrm{GmbH}$. All the specific reagents utilized for cell cultures were purchased from Sigma Aldrich and ATCC. The cell culture protocols were performed according to the manufacturer's specifications and as described in the literature [14-16].

Melanin was acquired from Sigma Aldrich and in order to obtain the standard melanin curves, the following protocol was applied: from the melanin stock solution - $2 \mathrm{mg} / \mathrm{mL}$ in $\mathrm{NH}_{4} \mathrm{OH}$ dilutions were prepared in the culture medium so that the final concentrations in the plate were: $0 ; 5 ; 10 ; 15 ; 20 ; 25$; 
30; 35; 40; 45; 50; 100; 150; 200 and $250 \mu \mathrm{g} / \mathrm{mL}$. Melanin solutions were applied to the 96-well plate in duplicate and absorbance was read at $405 \mathrm{~nm}$ using a spectrophotometer.

GraphPad Prism 6.0 statistical analysis software was used to determine the melanin concentration in the environment.

\section{Results and discussions}

\subsection{Determination of melanin content in the extracellular environment}

In order to determine the melanin content of the medium from the tested samples, it was necessary to obtain the standard melanin curves in different culture media specific to each cell line. The most used culture media in our laboratory for the growth of melanoma / melanocyte cell lines are: DMEM Dulbecco's Modified Eagle Medium, EMEM - Eagle Minimum Essential Medium, RPMI-1640, McCoy's 5A and Dermal Cell Basal Medium, and for these media standard curves were determined.

The standard curves for melanin and related equations were presented in the figure 1.

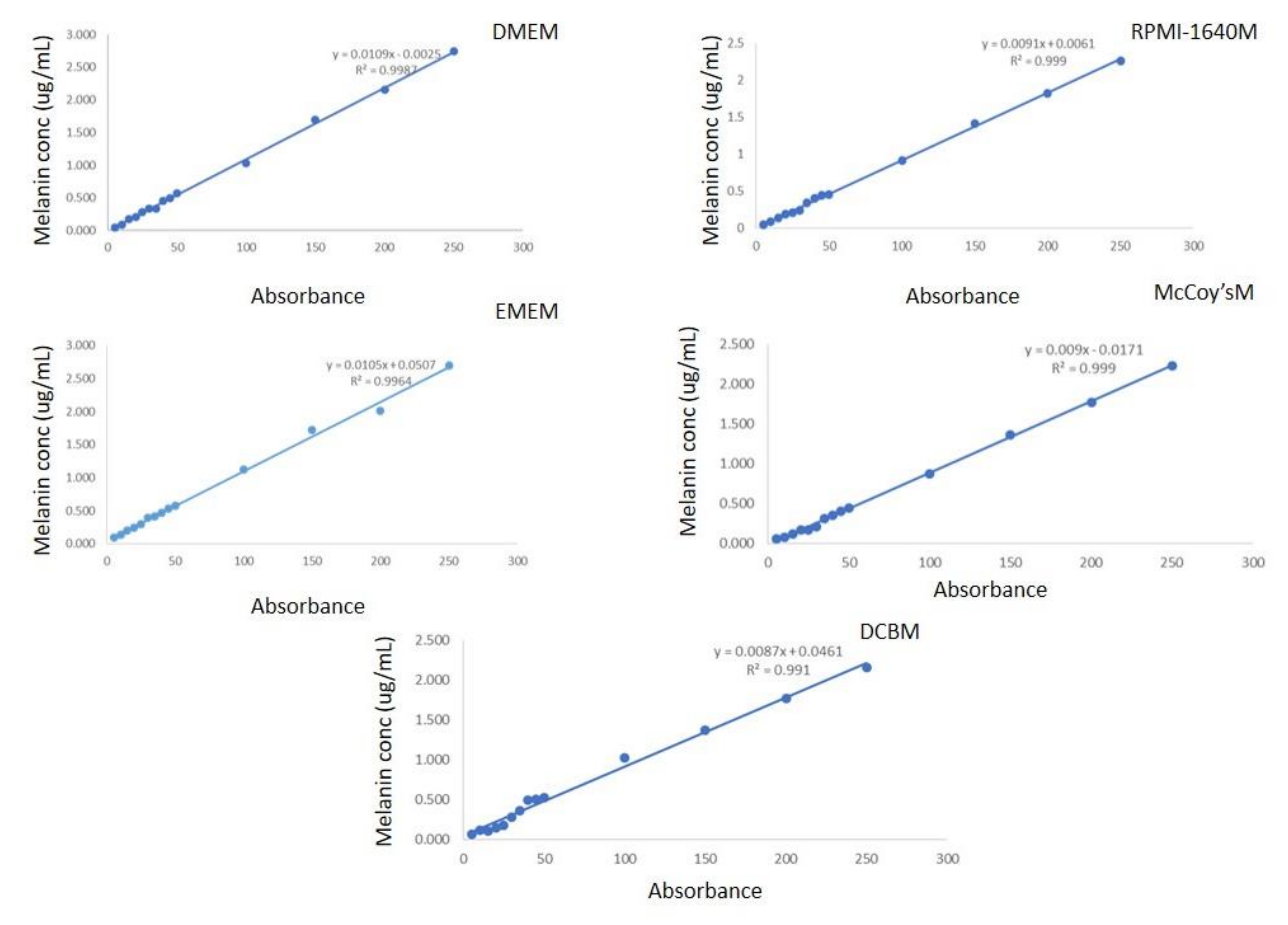

Figure 1. Standard curves for melanin and related equations in different specific medium.

\subsection{Melanin content in the cells}

The methodology employed to evaluate the melanin content from the extracellular environment, was conducted according to the protocol described by Yoo et al., 2007. [17] Briefly, the test cells (healthy or melanoma) were cultured in 96-well plates of culture and the main steps were: medium collection; culture centrifugation at $1200 \mathrm{x}$ g for $10 \mathrm{~min}$ and absorbance reading at $405 \mathrm{~nm}$. This protocol can also be applied if the cells are treated with different test compounds. For this experiment, the culture medium was collected from several healthy cell lines and melanoma cell lines of human and murine origin and using the standard curves the concentration of melanin in the environment was determined. The cell lines used in the study were: (i) human melanoma cell lines: A375, SK-MEL-1, SK-MEL-3, SK-MEL-5, SK-MEL-28, SH-4, and COLO 829; (ii) mouse melanoma cell lines: B164A5, B16F0, and B16F10; and (iii) healthy cell lines of human origin: HEMa (primary melanocytes), HaCaT (immortalized keratinocytes) and 1BR3 (human fibroblasts). In the experiment, which aimed to quantify the melanin content according to the cell line, but also according to the passage (especially those of murine origin) valuable data were obtained. Regarding human melanoma 
cell lines, the results are presented in Table 1.

Table 1. Melanin content in human melanoma cell lines spectrophotometrically determined

Cell line

$A 375$

SK-MEL-1

SK-MEL-3

SK-MEL-5

SK-MEL-28

SH-4

COLO 829
Cell morphology

Melanin content $(\mu \mathrm{g} / \mathrm{mL})$
6.358

3.704
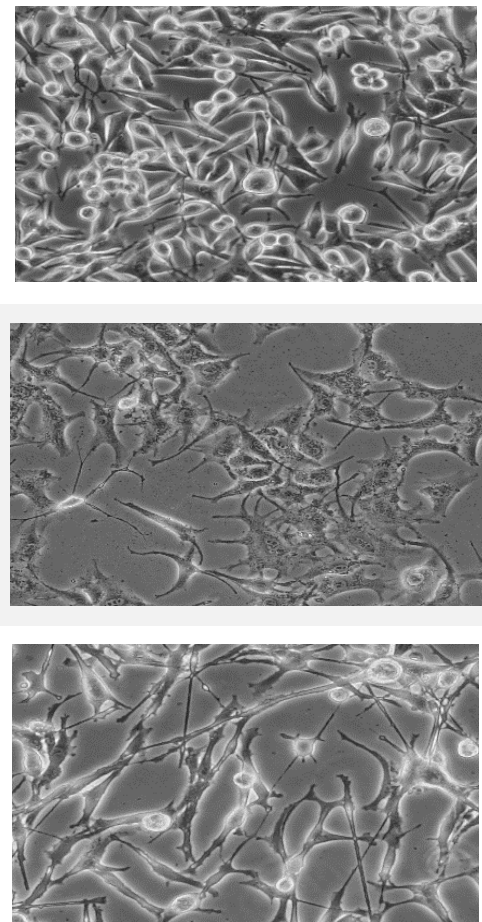

1.829

5.891

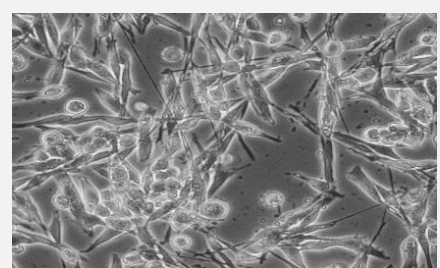


As can be noted A375, SK-MEL-28 and SK-MEL-5 cells do not produce melanin, SK-MEL-1, SH4, and SK-MEL-3 cells produce melanin while COLO 829 cells - in small passages produce melanin. Of the 3 lines of murine melanoma tested, the one that produces melanin in a higher concentration is $\mathrm{B} 16 \mathrm{~F} 10$, followed by B16F0 and B164A5 (see Table 2). In the case of B16F0 line, the longer the culture time and the number of passages increases, the lower the melanin concentration is present (data not shown).

Table 2. Melanin content in murine melanoma cell lines spectrophotometrically determined

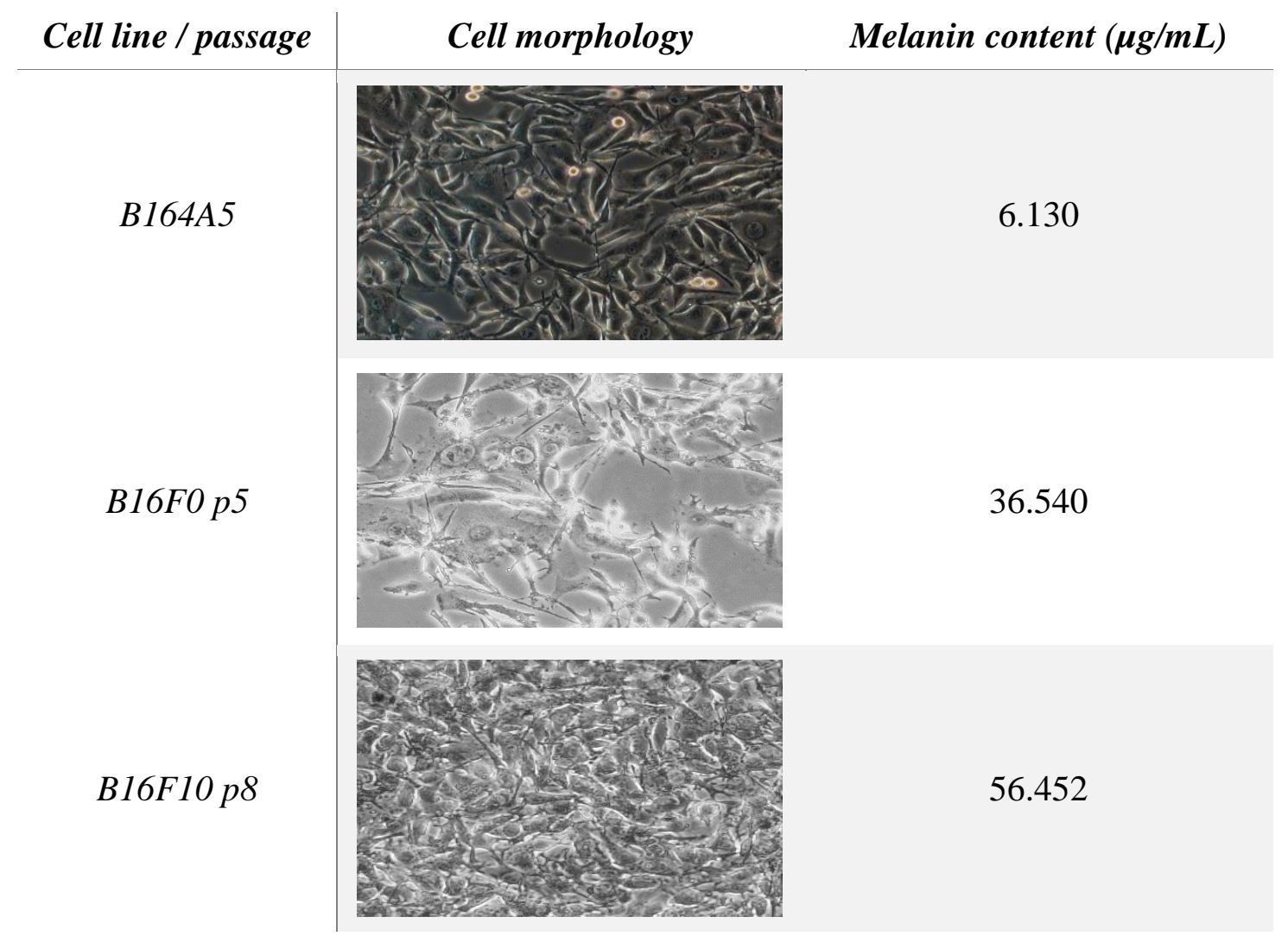

Regarding the healthy cell lines of human origin, melanin was detected in human melanocytes while in the case of keratinocytes and fibroblasts no melanin content was recorded (Table 3).

Due to the fact that melanocyte function is regulated by various autocrine and paracrine factors (factors that occur in skin cells such as keratinocytes, fibroblasts and melanocytes), it can be hypothesized that skin pigmentation associated with UV radiation is based on these factors [18]. A number of studies in the field have shown that the presence of melanin in melanoma decreases the photocytotoxicity exerted by drugs: in the case of photodynamic therapy, violet light was able to discolour melanin in melanotic tumours increasing the sensitivity to red light, the phototoxicity of hypericin is more pronounced in non-pigmented melanoma cells (e.g. A375 cells) compared to pigmented ones (e.g. UCT MEL-1 cells). It was also shown that 5-ALA and PDT with red light produced differential sensitivity in melanoma cells correlated with the presence of melanin, and mitochondrial apoptotic signalling was activated in pigmented B16F10 cells but not in unpigmented B16G4F cells. [19] Lu and co-workers have shown that adult human RPE cells do not produce melanin in vitro correlated with the fact that the melanin content of these cells decreases markedly in the aging process and may be related to the appearance of different diseases of the retina; on the other hand, human uveal melanoma cells produce melanin in vitro, expressing melanogenic genes, which may be considered for in-depth studies in understanding the mechanisms of melanoma, assuming that they may have a similar pathway for melanin biosynthesis (such as cutaneous melanocytes and 
Table 3. Melanin content in human healthy cell lines spectrophotometrically determined

\begin{tabular}{|c|c|c|}
\hline Cell line & Cell morphology & Melanin content $(\mu \mathrm{g} / \mathrm{mL})$ \\
\hline$H E M a$ & & 3.879 \\
\hline $1 B R 3$ & & - \\
\hline $\mathrm{HaCaT}$ & & - \\
\hline
\end{tabular}

melanoma cells). [20] In a previous study, it was noted that exposure to UVB radiation of melanocytes, and two other types of human melanoma cells (SK-MEL-3 and COLO-829), led to harmful effects on both melanocytes and melanoma cells, the most affected being COLO-829 cells, whereas at $24 \mathrm{~h}$ post-exposure the melanocytes recovered completely. [16] From the data obtained in the present study it can be observed that melanocytes and SK-MEL-3 cells have a similar melanin content while COLO-829 cells have a higher melanin content. In future studies, the effect of type A and type $\mathrm{B}$ radiation on the cells from this study will be assessed.

\section{Conclusions}

The skin, the largest organ of the human body, contains an excellent pigment - melanin - which has the ability to cushion the effect of ultraviolet radiation by absorbing it, but also the ability to repair the mechanisms related to DNA damage induced by UV radiation. At the same time, pigmentation is an essential factor that can significantly contribute to reducing the incidence of melanoma and other types of skin cancers. Melanoma skin cancer is one of the most therapy-resistant types of cancer and even with proper chemotherapy the prognosis is extremely poor. Currently, many studies target tyrosinase, the enzyme that synthesizes melanin, unique to melanogenic cells, as a promising anti-melanoma target, and efforts for this approach are still ongoing. Therefore, the data obtained regarding the melanin content in different cell lines, offer an essential background for subsequent studies involving tests for anti-melanoma potential of the new candidates.

Funding: This study was financially supported by a grant of the Romanian National Authority for Scientific Research and Innovation, CNCS-UEFISCDI, project no. PN-III-P1-1.1-PD-2016-1982.

Acknowledgments: The experiments were conducted within the Center of Pharmaco-toxicological evaluations from the Faculty of Pharmacy, "Victor Babes" University of Medicine and Pharmacy, Timisoara. 


\section{References}

1. BRENNER, M., HEARING, V.J. The Protective Role of Melanin Against UV Damage in Human Skin, Photochem Photobiol., 84, no.3, 2008, 539-549.

2. TRAN, T.N., SCHULMAN, J., FISCHER, D.E. UV and pigmentation: molecular mechanisms and social controversies, Pigment Cell Melanoma Res., 21, no. 5, 2008, 509-516.

3. CHOI, W., MiYAMURA, Y., WOLBER, R., SMUDA, C., REINHOLD, W., LIU, H., et al. Regulation of Human Skin Pigmentation in situ by Repetitive UV Exposure - Molecular Characterization of Responses to UVA and/or UVB, J Investig Dermatol. 130, no. 6, 2010, p. 16851696.

4. SCHLENZ, K., SMUDA, C., BATZER, J. et al. Pigmentation mechanisms induced by different wavelengths of UV light., Pigment Cell Res., 18, 2005, S33

5. MABUlA, J.B., CHALYA, P.L., MCHEMBE, M.D., JAKA, H., GIITI, G., RAMBAU, P., et al. Skin Cancers Among Albinos at a University Teaching Hospital in Northwestern Tanzania: A Retrospective Review of 64 Cases, BMC Dermatol., 12, 2012, 5

6. MOHANIA, D., CHANDEL, S., KUMAR, P., VERMA, V., DIGVIJAY, K., DEEPIKA TRIPATHI, D. et al. Ultraviolet Light in Human Health, Diseases and Environment, Advances in Experimental Medicine and Biology, 2017, p. 71.

7. MARINCU, I., FRENT, S., TOMESCU, M.C., MIHAICUTA, S. Rates and predictors of uncontrolled bronchial asthma in elderly patients from western Romania, Clin Interv Aging. 10, 2015, 963-967.

8. TAlPOS, S., HAJAJ, T., TIMOFTE, C., RIVIS, M., STREIAN, F., POPA, M. et al. Implant surgery using bio-compatible guides, Mater. Plast. 55, (1), 2017, 38-41.

9. MUNTEANU, M.F., ARDELEAN, A., BORCAN, F., TRIFUNSCHI, S.I., GLIGOR, R., ARDELEAN, S.A. et al. Mistletoe and Garlic Extracts as Polyurethane Carriers - A Possible Remedy for Choroidal Melanoma, Curr Drug Deliv. 14, no. 8, 2017, 1178-1188.

10. DUICU, O., CIURLEA, S., DEHELEAN, C., ARDELEAN, S., ANDRICA, F., MUNTEAN, D. et al. Analysis of a betulinic acid formulation on liver mitochondria isolated from mice with induced murine melanoma, Rev Chim., 65, (8), 2014, 956-959.

11. CORINA, D., FLORINA, B., IULIA, P., CRISTINA, D., RITA, A., ALEXANDRA, P. et al. Rutin and Its Cyclodextrin Inclusion Complexes: Physico-chemical Evaluation and in Vitro Activity on B164A5 Murine Melanoma Cell Line, Curr Pharm Biotechnol., 18, no. 13, 2017, 1067-1077.

12. ANDOR, B., TISCHER, A.A., BERCEANU-VADUVA, D., LAZUREANU, V., CHEVERESAN, A., POENARU, M. Antimicrobial Activity and Cytotoxic Effect on Gingival Cells of Silver Nanoparticles Obtained by Biosynthesis, Rev Chim., 70, (3), 2019, 781-783.

13. BROŻYNA, A.A., JÓŹWICKI, W., ROSZKOWSKI, K., FILIPIAK, J., SLOMINSKI, A.T. Melanin content in melanoma metastases affects the outcome of radiotherapy, Oncotarget, 7, no. 14, 2016, 17844-17853.

14. GYORI, Z., SUSAN, M., SUSAN, R., IFTODE, A., TRANDAFIRESCU, C., CHEVERESAN, A., et al. Melanoma cell lines role in obtaining new drug candidates for combating the malignant pathology of the cutaneous organ, Rev Chim., 70, (3), 2019, 943-945.

15. CORICOVAC, D., FARCAS, C., NICA, C., PINZARU, I., SIMU, S., STOIAN, D., et al. Ethinylestradiol and Levonorgestrel as Active Agents in Normal Skin, and Pathological Conditions Induced by UVB Exposure: In Vitro and In Ovo Assessments, Int J Mol Sci., 19, no.11, 2018, 3600.

16. GYORI, Z., BERCEANU, M., CHEVERESAN, A., FARCAS, C., BRATU, T., CRAINICEANU, Z. Melanin and Behaviour of Melanoma-related Cell Lines in the Presence of Ultraviolet B Type Radiation, Rev Chim., 70, (7), 2019, 2398-2400.

17. YOO, B.-S., YOO, M.A., SONG, Y.K., BYUN, S.Y. Regulation of proteins related to melanogenesis by heartwood extract ofMorus bombycis using proteome analysis, Biotechnol. Bioprocess Eng., 12, 2007, 662. 
18. YAMAGUCHI, Y., BRENNER, M., HEARING, V.J. The Regulation of Skin Pigmentation, J Biol Chem., 282, no. 38, 2007, 27557.

19. SPARSA, A., BELLATON, S., NAVES, T., JAUBERTEAU, M.-O., BONNETBLANC, J.-M., VERDIER, V.S.M., RATINAUD, M.-H. Photodynamic treatment induces cell death by apoptosis or autophagy depending on the melanin content in two B16 melanoma cell lines, Oncol. Rep., 29, no. 3, 2013, 1196-1200.

20. LU, F., YAN, D., ZHOU, X., HU, D.-N., QU, J. Expression of melanin-related genes in cultured adult human retinal pigment epithelium and uveal melanoma cells, Mol Vis. 13, 2007, 2066-2072.

Manuscript received: 12.11 .2019 